\title{
THEORY OF GOLD-HYDROGEN COMPLEXES IN SILICON
}

\author{
A Resende ${ }^{1}, \mathrm{~J} \mathrm{Goss}^{1}, \mathrm{P}$ R Briddon ${ }^{2}, \mathrm{~S}$ Öberg $^{3}$ and R Jones ${ }^{1}$ \\ 1 Department of Physics, The University of Exeter, Exeter, EX4 4QL, United Kingdom \\ 2 Department of Physics, The University of Newcastle upon Tyne, \\ Newcastle upon Tyne, NE1 7RU, United Kingdom \\ 3 Department of Mathematics, University of Luleå, Luleå, S-97187, Sweden
}

Keywords: $a b$ initio theory, Si, Au, H, G complex, passivation.

\begin{abstract}
Spin-polarised local density functional cluster calculations are carried out on substitutional Au and $\mathrm{A} u-\mathrm{H}_{n}$ complexes in Si. Slater's transition argument and Janak's theorem are combined to discuss the donor-acceptor levels of the defects and the results are compared with experiment. The calculations give a good account of the donor/acceptor levels of $\mathrm{Au}$ and the $\mathrm{Au}-\mathrm{H}$ pair. They confirm that the G1, G2 and G4 levels are due to $\mathrm{Au}-\mathrm{H} . \mathrm{Au}-\mathrm{H}_{3}$ is found to be an electrically inactive defect and may be identified with the passive defect found experimentally.
\end{abstract}

\section{Introduction}

Transition metals are important impurities in Si as they possess deep levels in the bandgap and interfere with the properties of devices. For example, they reduce storage time in MOS dynamical memory cells and lead to the formation of dark current spikes in CCDs.

Gold as an isolated impurity in silicon is one of the most studied defects in semiconductors. This is mainly due to its technological important and to its pivotal role in the understanding of the electronic properties of transition metal impurities in semiconductors. Elements like gold, platinum, silver or even titanium, are commonly used to produce metallic contacts. Our understanding of the impurities at the far right of the periodic table is based on the "vacancy model" [1]. This maintains that their electronic activity is controlled by the levels of the vacancy. Since, the vacancy can be passivated [2], there is a possibility that the transition metal impurity can also be passivated and this has been found in the case of $\mathrm{Au}$ [3].

\section{Background experimental results}

The amphoteric behaviour of gold in silicon has been a matter of controversy for many years. Only recently [4] has it been unequivocally established that substitutional gold introduces two levels in the band gap. These deep acceptor and donor levels lie at $E_{c}-0.54$ and $E_{v}+$ $0.35 \mathrm{eV}$ respectively. The ground state of the substitutional $\mathrm{Au}^{0}$ impurity has $C_{2 v}$ symmetry, tetragonally distorted, spin state $\mathcal{S}=1 / 2$ and configuration $b_{2}^{\uparrow \downarrow} b_{1}^{\uparrow} a_{1}^{0}[5]$, with $g_{\|} \approx 2.8$ and $g_{\perp} \approx 0$. The distortion is in agreement with the predictions of the vacancy model. The defect can reorientate easily at cryogenic temperatures $(T<4 \mathrm{~K})[6]$ suggesting a very small departure from $T_{d}$ symmetry. Surprisingly, no EPR signal has been attributed to the defect and this is understood to arise by a rapid tunnelling between two equivalent $C_{2 v}$ configurations [7] driven by a dynamical Jahn-Teller effect. This makes the $g_{x x}$ and $g_{y y}$ components of the $g$ tensor very small and hence the microwave $\Delta m_{J}= \pm 1$ transitional probability becomes very low and the 
EPR spectrum difficult to observe. This model is supported by recent theoretical work using the same methods as those described here [8]. The theory finds that $\mathrm{Au}^{0}$ distorts along the [100] direction by $\sim 0.18 \AA$ with small shifts of the neighbouring $\mathrm{Si}$ atoms. The barrier to the reorientation is then only $\sim 0.01 \mathrm{eV}$ and the defect might be expected to tunnel easily between symmetric configurations.

We concentrate in this paper on the energy levels of the defect and the effect of $H$ upon them. The first study on the influence of $\mathrm{H}$ on the gold centre was reported by Pearton and Tavendale [3] using samples exposed to a plasma between 150 and $350^{\circ} \mathrm{C}$. A substantial loss of the Au activity was reported which could, however, be partially reactivated by annealing at $400^{\circ} \mathrm{C}$. No effect was found for samples annealed in hydrogen gas.

Wet-chemical etching is another process commonly used to introduce hydrogen into the samples. This process has some advantages over the the use of a plasma. H is introduced at room temperature, without any damage to the wafer surface, and leads to the formation of $\mathrm{Au}-\mathrm{H}$ defects which posses distinct deep levels [9]. These defects disappear around $\sim 150^{\circ} \mathrm{C}$ and are transformed to a passive defect if sufficient $H$ is available. Such $H$ could be released from the dissociation of P-H defects. The passive defect is destroyed by annealing beyond $200^{\circ} \mathrm{C}$. This temperature is lower than that found by Pearton and Tavendale, probably because they heated the sample in the presence of the plasma, which provided a continuous source of $H$. $\mathrm{Pt}-\mathrm{H}_{2}$ defects appear to be more stable, forming around $\sim 400^{\circ} \mathrm{C}$.

The Au-H defects formed in wet-chemical etching have been studied by Sveinbjörnsson et al [10]. Four new deep levels named G1-G4 were observed in the DLTS spectra. G1 is an acceptor level observed in $n$-type material with an activation energy of $0.19 \mathrm{eV}$. G2 and G3 are hole traps detected in $p$-type $\mathrm{Si}$ and located at $E_{v}+0.21$ and $E_{v}+0.47 \mathrm{eV}$ respectively. G4 was detected in both $n$ - and $p$-type samples and showed similar characteristics to those of the gold acceptor level. It was suggested that G1, G2 and G4 are levels of the same Au-H defect, probably Au complexed with a single $\mathrm{H}$ atom, but the PA complex contained additional $\mathrm{H}$ atoms. However, the DLTS study did not reveal the number of $\mathrm{H}$ atoms in the defects. Little is known about the G3 defect.

A very recent study on the electron- and hole-capture kinetics of $\mathrm{Au}-\mathrm{H}$ complexes in Si has been carried out using Minority Carrier Transient Spectroscopy (MCTS) [11]. This technique, combined with DLTS, allowed the determination of acceptor and donor levels using only one type of material and is a useful technique for defects with multiple levels. The main conclusion refers to the nature of the G1, G2 and G4 levels. Davidson and Evans confirmed that these levels are all produced by the same Au-H complex but G1 was shown to be a double acceptor level.

\section{Method}

Ab initio spin-polarised local density functional cluster theory (AIMPRO) was used to explore the properties of $\mathrm{Au}-\mathrm{H}$ defects. Full details of the technique have been given before and will not be repeated here [12]. Large tetrahedral H-terminated clusters, containing 131 and 297 atoms with the configurations $\mathrm{Si}_{71} \mathrm{H}_{60}$ and $\mathrm{Si}_{181} \mathrm{H}_{116}$ respectively, were used in this investigation. The basis consisted of $N$ Cartesian $s, p$ Gaussian orbitals sited on each atom. $N$ independent $d$ orbitals were sited on the Au atom. The charge density is fitted to $M$ Gaussian functions. In this study, $(N, M)$ are: $\mathrm{Au}(6,12), \mathrm{Si}(4,5)$ and $\mathrm{H}(2,3)$. The pseudo-wave functions were expanded in independent combinations of the $N$ Gaussian orbitals on atoms at the centre of the cluster but a fixed linear combination of the $N$ orbitals was used on those outside the defect core. The pseudopotentials were those of Bachelet et al. These were placed on all atoms except H. The 
self-consistent energy was found together with the analytic forces on each atom. The structure was then relaxed using a conjugate gradient algorithm.

This method has been successfully applied to the study of $\mathrm{Ni}$ and $\mathrm{Ni}-\mathrm{H}$ complexes in silicon [13] as well as the ferrocene molecule [14] and $\mathrm{Ni}$ defects in diamond [15].

Of particular interest here are the donor and acceptor energy levels associated with the defects. But there are several problems relating to the formalism in extracting these levels. The acceptor or $(-/ 0)$ level, relative to the conduction band, is simply the energy difference between a negatively charged and neutral defect with an additional electron in the lowest conduction band state. Suppose $d$ is the Kohn-Sham energy level of the highest occupied level associated with the ionised defect and let $c$ be the Kohn-Sham level associated with the conduction band minimum. Now, provided the relaxation of the defect when the electron is promoted between these levels can be ignored, the difference in the energies of these configurations is, according to Slater's transition state argument and Janak's theorem, simply the difference in the $d$ and $c$ levels found for an electronic configuration corresponding to half-occupancy, i.e, one-half a electron is removed from the level $d$ and placed in level $c$. If this were the only approximation, the errors would be very slight. Unfortunately, density functional theory is not strictly applicable to the excited state configuration and as such band gaps are substantially underestimated. However, the use of a H-terminated cluster often leads to a band gap which exceeds the experimental one and is a consequence of the large confining potential exerted on the cluster by the terminating $\mathrm{H}$ atoms. In diamond, these two effects tend to cancel with the result that the gap is fortuitously in agreement with the experimental gap. The use of the Slater scheme to calculate excitation energies then leads to values in reasonable agreement with known optical transitions [16]. Unfortunately, in silicon the cluster band gaps are between a factor three and four too large. To deal with this problem, we have chosen to scale the Slater's transition state energies by the ratio of the correct Si band gap and the cluster gap. This has the effect of severely reducing all the donor-acceptor levels. In an analogous way, donor levels can be found by promoting half a electron from the valence band to the lowest unoccupied defect level corresponding to the positively charged defect. The technique is easily extended to second acceptor or donor levels. While the approach is not completely satisfactory, it does lead to levels in known cases which are within $0.2-0.3 \mathrm{eV}$ of experiment, although it appears that the levels found are deeper than the experimental ones.

\section{The substitutional Au centre}

An 131 atom cluster with a neutral Au placed at its centre was fully relaxed, keeping the $\mathrm{H}$ terminators fixed. The gap contains a $t_{2}$ level containing three electrons and the configuration is taken to be $t_{2}^{\uparrow \uparrow} t_{2}^{\downarrow}$, consistent with $\mathcal{S}=1 / 2$. The charge density was averaged over the three degenerate $t_{2}$ states and the self-consistent energy and forces acting on the atoms found. This procedure prevents a Jahn-Teller distortion [13]. Such distortions have been investigated by breaking the symmetry and relaxing in a specific electronic configuration [8, 13] but the $t_{2}$ splitting is very small and of no importance to the calculation of the donor and acceptor levels. The Au-Si lengths were $2.76 \AA$.

The positions of the five innermost atoms, including the impurity, were then inserted into a 297 atom cluster. An additional half electron was added to the $t_{2}^{\downarrow}$ level and another half added to the nearest $a_{1}$ empty level which is taken to be the conduction band bottom. The difference between these levels then gave an estimate of the $(-/ 0)$ acceptor level. In a similar way, the donor level was found by promoting half a electron to the $t_{2}$ level of the positively charged defect from the three levels lying beneath this defect level (and taken to represent thee valence band top). These energies were then rescaled as described above to compensate for the cluster 
band gap. With the present basis, the band gap in the 297-atom cluster without any impurities is $3.51 \mathrm{eV}$. The scaling factor is then 0.33 and the resulting estimates for the levels are given in Table 1.

The deep acceptor level at $E_{c}-0.63 \mathrm{eV}$ compares well with the experimental value of 0.54 $\mathrm{eV}$. The donor level at $E_{v}+0.45 \mathrm{eV}$ is deeper than than the experimental value of $0.35 \mathrm{eV}$. A second acceptor level lay at $E_{c}-0.017 \mathrm{eV}$. There is no evidence for this shallow level and it presumably lies above the conduction band minimum. The sum of the $(0 /+)$ and $(-/ 0)$ levels is $1.08 \mathrm{eV}$ which is almost equal to the energy gap $1.16 \mathrm{eV}$, whereas the experimental sum is $0.89 \mathrm{eV}$. The latter differs from the gap because of the Hubbard $U$ term. It is clearly a failing of the present theory to account for this term properly.

Table 1. Comparison between experimental $[10,11]$ and calculated energy levels $\Delta E$ for the gold centre and $\mathrm{Au}-\mathrm{H}$ complex (G complex).

\begin{tabular}{|cccc|}
\hline Defect & Peak & Calc. (eV) Expt. (eV) \\
\hline \hline \multirow{3}{*}{$\mathrm{Si}: \mathrm{Au} u^{0}$} & $\mathrm{Au}(-2 /-)$ & 0.017 & - \\
& $\mathrm{Au}(-/ 0)$ & 0.63 & 0.54 \\
& $\mathrm{Au}(0 /+)$ & 0.45 & 0.35 \\
\hline \multirow{3}{*}{$\mathrm{G} 1(-2 /-)$} & 0.36 & 0.19 \\
$\mathrm{Au}-\mathrm{H}$ & $\mathrm{Au}(-/ 0)+\mathrm{G} 4$ & 0.63 & midgap \\
& $\mathrm{G} 2(0 /+)$ & 0.39 & 0.21 \\
\hline
\end{tabular}

\section{$\mathrm{Au}-\mathbf{H}_{n}$}

We investigated the $\mathrm{Au}-\mathrm{H}_{n}$ complexes when $\mathrm{H}$ lies both at anti-bonding sites outside the nearest neighbour shell of Si atoms to the impurity, and inside the shell. Similar sized clusters were used as in the Au defect discussed above.

For the $\mathcal{S}=0 \mathrm{Au}-\mathrm{H}$ defect, the $\mathrm{H}$ atom lies near a bond centred site as illustrated in Fig. 1. This configuration is more stable than the AB one by $\sim 0.2 \mathrm{eV}$. The Au-H and H-Si lengths are 1.78 and $1.57 \AA$ respectively with an $\mathrm{Au}-\mathrm{H}-\mathrm{Si}$ angle of $118^{\circ}$. The difference in energy between a configuration where the $\mathrm{H}$ atom is constrained to lie along [100] with $C_{2 v}$ symmetry is $0.15 \mathrm{eV}$ and this represents an estimate of the energy barrier to reorientation.

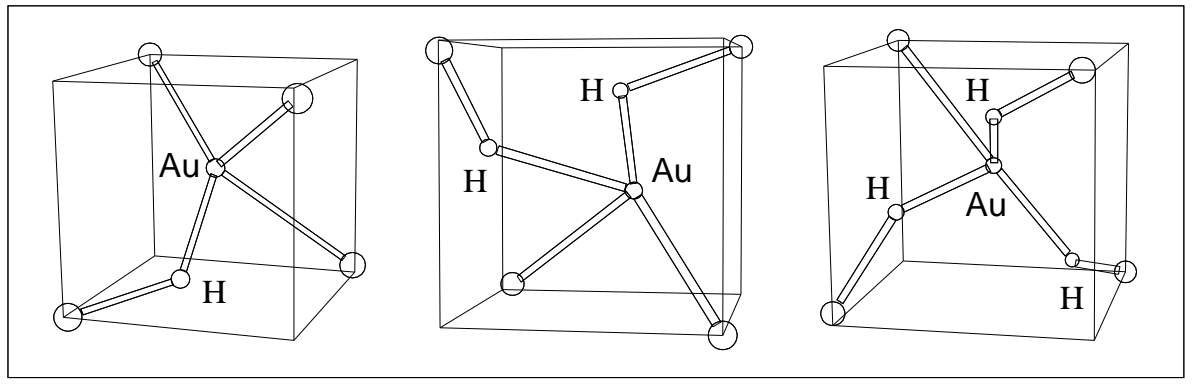

Fig. 1. Relaxed structure of the $\mathrm{Au}_{\mathrm{Si}}-\mathrm{H}_{n}$ in $\mathrm{Si}, n=1,2$ and 3. Box edges mark the $\{100\}$ directions. Note the increasing $\measuredangle(\mathrm{Au}-\mathrm{H}-\mathrm{Si})$ with increasing number of $\mathrm{H}$ atoms.

The $t_{2}$ level (Fig. 2) now contains four electrons and is split and displaced downwards in the gap by the proton. Inevitably, this will decrease the donor and deepen the acceptor levels - as observed for G2 and G4 - , and encourages the possibility of a second acceptor level. The Slater method locates the acceptor $(-/ 0)$ level at $0.63 \mathrm{eV}$ which is the same as that of Au. This agrees with the assignment of the mid-gap G4 level. A second acceptor $(-2 /-)$ level is found at $E_{c}-0.36 \mathrm{eV}$ and about $0.17 \mathrm{eV}$ deeper that the $(-2 /-) \mathrm{G} 1$ level. The donor $(0 /+)$ level at $E_{v}+0.39 \mathrm{eV}$ is slightly lower than that of $\mathrm{Au}$ at $E_{v}+0.45 \mathrm{eV}$ and is $0.17 \mathrm{eV}$ above 
G2. Given that the levels are generally deeper than about $0.1-0.2 \mathrm{eV}$, the results support the finding that the G1, G2, G4 levels all arise from the same defect which contains just one H atom. In addition, they support the claim that the $\mathrm{G} 3$ level at $E_{v}+0.47 \mathrm{eV}$ cannot arise from this defect.

Table 2. Bond lengths for the different complexes. The bottom row of the table gives the distance $(d)$ of the Au atom from the centre of the cluster. Bond lengths in $\AA$.

\begin{tabular}{|c|c|c|c|}
\hline Bond $\mathrm{Au}^{0}$ & $\mathrm{Au}-\mathrm{H}$ & $\mathrm{Au}-\mathrm{H}_{2}$ & $\mathrm{Au}-\mathrm{H}_{3}$ \\
\hline \multicolumn{3}{|c|}{ 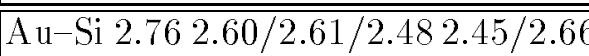 } & 2.45 \\
\hline $\mathrm{Au}-\mathrm{H}-$ & 1.78 & $1.72 / 1.81$ & $4 / 1.84 / 1.70$ \\
\hline $\mathrm{H}-\mathrm{Si}$ & 1.57 & $1.53 / 1.6$ & $7 / 1.50 / 1.50$ \\
\hline$d$ & 0.26 & 0.63 & 0.60 \\
\hline
\end{tabular}

Adding additional $\mathrm{H}$ atoms to the defect will fill the $t_{2}$ level and shift it downwards (Fig. 2). The level becomes filled for $\mathrm{Au}-\mathrm{H}_{3}$. The $\mathrm{H}$ atoms prefer to be inside the vacancy as the energy of this structure is $0.8 \mathrm{eV}$ below that of the outside configuration. Two $\mathrm{H}$ atoms are strongly bonded to the Si radicals with lengths of $1.50 \AA$ whereas the third is $1.67 \AA$ from a third radical and 1.64 $\AA$ from Au. The gold atom is displaced off-site by $\sim 0.6 \AA$ along [100]. The defect has $\mathcal{S}=0$ and the filled Kohn-Sham levels now are low lying and there are no empty gap levels. Thus the defect cannot act as an acceptor. The donor level as calculated by Slater's method is placed at $E_{v}+0.18 \mathrm{eV}$. Since our method leeds to deeper levels by $\sim 0.2 \mathrm{eV}$ than observed, the defect may identify $\mathrm{Au}-\mathrm{H}_{3}$ with the passive $\mathrm{PA}$ complex. This suggests that the Au-H defect is passivated by a direct combination with a mobile hydrogen molecule.

The $\mathrm{Au}-\mathrm{H}_{2}$ and $\mathrm{Au}-\mathrm{H}_{4}$ defects are electrically and magnetically active but will not be discussed here. It seems unlikely that the G3 level is one of these defects.

Fig. 2. One-electron Kohn-Sham eigenvalues in the vicinity of the gap for the $\mathrm{Au}_{\mathrm{Si}^{-}}-\mathrm{H}_{n}$ complexes in their relaxed configurations, with $n=1, \cdots, 4$. Arrows indicate filled spin states, boxes indicate empty one-electron states (these are only shown in region of the gap).

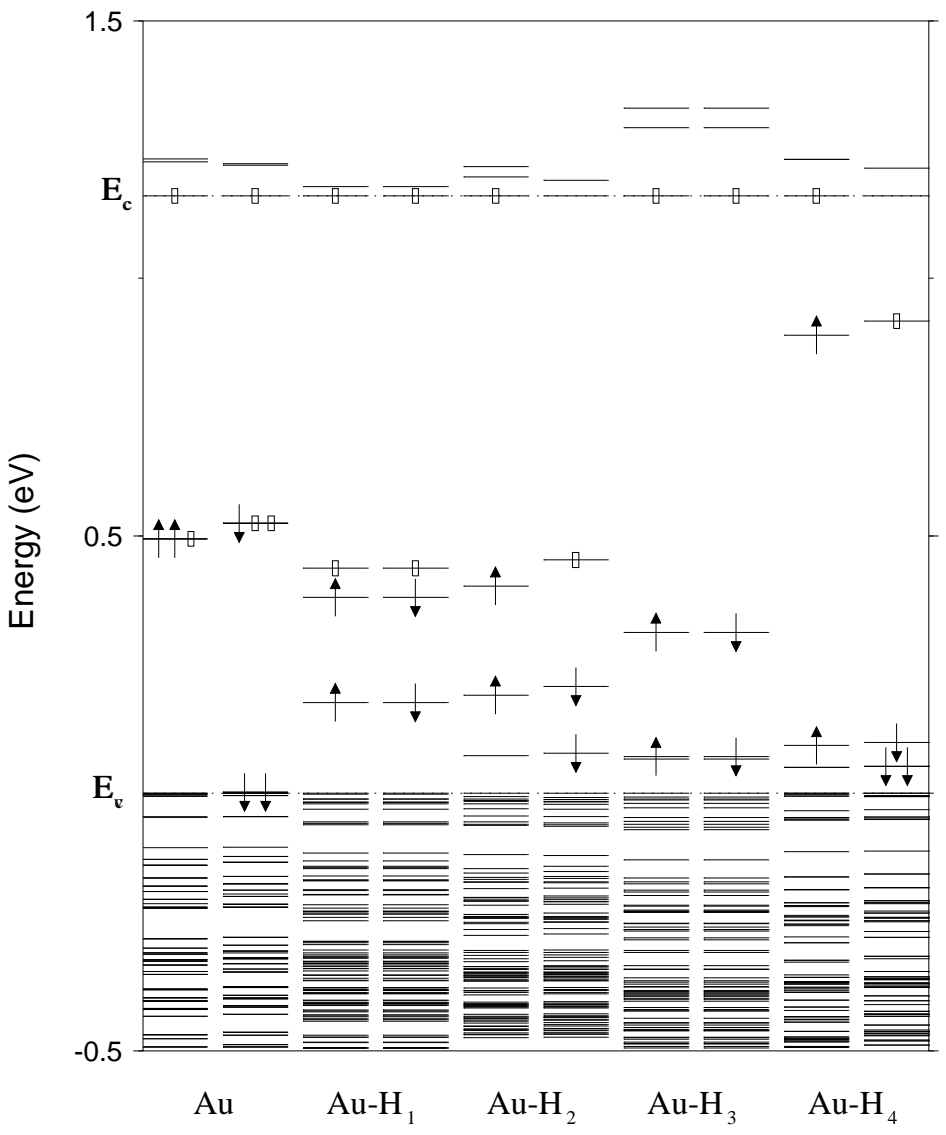

In conclusion, our calculations have found:

1. The substitutional Au centre has $(0 /+)$ and $(0 /-)$ levels within $0.2 \mathrm{eV}$ of the experimental values and a second acceptor level lies close to (and probably in) the conduction band; 
2. The Au-H complex is diamagnetic and more stable with $\mathrm{H}$ close to $\mathrm{Au}$ near a bond centred configuration. The reorientation energy for $\mathrm{Au}$ is close to $0.15 \mathrm{eV}$;

3. The Au-H defect has deeper acceptor and more shallow donor levels than Au. We find the $(-2 /-),(0 /-),(0 /+)$ levels within $0.2 \mathrm{eV}$ of those of G1, G2 and G4 which are associated with one defect;

4. Two further $\mathrm{H}$ atoms add to Au-H by saturating two of the Si dangling bonds. The defect is diamagnetic;

5. Au- $\mathrm{H}_{3}$ has no acceptor levels and a shallow donor level. We identify it with the passive defect first detected by Pearton and Tavendale;

6. Au- $\mathrm{H}_{2}$ and $\mathrm{Au}-\mathrm{H}_{4}$ are electrically and magnetically active defects with deep levels.

\section{Acknowledgements}

S Öberg thanks NFR and TFR in Sweden for financial support. He also thanks PDC at KTH in Sweden, for computer time on the SP2. We also thank the HPCI committee of the EPSRC for computer time on the CRAY T3D where most of these results were derived.

\section{References}

[1] G D Watkins, Physica B117-B118, 9 (1983).

[2] B. Bech Nielsen, L. Hoffmann, M. Budde, R. Jones, J. Goss, and S. Öberg, Materials Science Forum 196-201, 933, (1995).

[3] S J Pearton and A J Tavendale, Phys. Rev B26, 7105 (1982).

[4] J W Petersen and J Nielsen, Appl. Phys. Lett. 56, 1122 (1990).

[5] M Kleverman, A Thilderkvist, G Grossman, H G Grimmeiss and G D Watkins, Sol. State Commun. 93, 383 (1983).

[6] G D Watkins and M Kleverman, Phys. Rev. Lett. 67, 1149 (1990).

[7] G D Watkins and P M Williams, Phys. Rev. B52, 16575 (1995).

[8] P R Briddon (To be published).

[9] W Jost and J Weber, Phys. Rev B54, 11038 (1996); W Jost, J Weber and H Lemke, Semicond. Sci. Technol. 11, 22 (1996).

[10] E Ö Sveinbjörnsson and O Engström, Phys. Rev. B52, 4884 (1995).

[11] J A Davidson and J H Evans, Semicond. Sci. Technol. 11, 1704 (1996).

[12] To appear in Identification of Defects in Semiconductors, edited by M Stavola and Semiconductors and Semimetals, treatise editors, R. K. Willardson, A C Beer and E R Weber (Academic Press).

[13] R Jones, S Öberg, J Goss, P R Briddon and A Resende, Phys. Rev. Lett. 75, 2734 (1995).

[14] A Resende, R Jones, S Öberg, P R Briddon and B R Eggen (unpublished)

[15] J Goss, A Resende, R Jones, S Oberg and P R Briddon, Materials Science Forum 196-201, 67$72,(1995)$.

[16] J P Goss, R Jones, S J Breuer, P R Briddon and S Öberg, Phys Rev Lett 77, 3041 (1996).

This article was processed using the IATEX macro package with TTP style 